\title{
Medical Image Segmentation using Modified K Means Clustering
}

\author{
Kalpana Shrivastava \\ M.Tech, Scholar, TIEIT, \\ Bhopal, India
}

\author{
Neelesh Gupta \\ HOD, ECE, TIEIT, Bhopal
}

\author{
Neetu Sharma \\ A.P., ECE, TIEIT
}

\begin{abstract}
:
Image segmentation is an important technique for image processing which aims at partitioning the image into different homogeneous regions or clusters. Lots of general-purpose techniques and algorithms have been developed and widely applied in various application areas. For the study of anatomical structures and to identify the region of interest. Magnetic Resonance Images are used to produce images of soft tissue of human body. Noise present in the Brain MRI images are multiplicative noise and reductions of these noise are difficult task. However, accurate Segmentation of the MRI images is very important and crucial for the exact diagnosis by computer aided clinical tools. A large variety of algorithms for segmentation of MRI images had been developed. However most of these have some limitations, to overcome these limitations; modified $\mathrm{k}$ means clustering is proposed. The comparison of existing segmentation approaches such as C-Means Clustering, K-Means Clustering with Modified KMeans Clustering is performed then the performance evaluated. Finally generated outcomes of the Fuzzy c- means clustering, k-means clustering and modified $\mathrm{k}$ means clustering algorithm for the brain MRI shows that modified kmeans clustering technique gives better results for all performance measuring parameters such as structural similarity index measure, structural content, mean squared error and peak to signal noise ratio.
\end{abstract}

\section{Keywords:}

Fuzzy C-Means, K-Means, SSIM, SC, PSNR

\section{INTRODUCTION}

Image segmentation was, is and will be a major research topic for many image processing researchers. The reasons are obvious and applications endless: most computer vision and image analysis problems require a segmentation stage in order to detect objects or divide the image into regions which can be considered homogeneous according to a given criterion, such as colour, motion, texture, etc. Clustering is the search for distinct groups in the feature space. It is expected that these groups have different structures and that can be clearly differentiated. The clustering task separates the data into number of partitions, which are volumes in the n-dimensional feature space. These partitions define a hard limit between the different groups and depend on the functions used to model the data distribution.

\subsection{Magnetic Resonance Imaging}

A magnetic resonance imaging (MRI) scanner uses powerful magnets to polarise and excite hydrogen nuclei (single proton) in human tissue, which produces a signal that can be detected and it is encoded spatially, resulting in images of the body. The MRI machine emits radio frequency (RF) pulse that specifically binds only to hydrogen. The system sends the pulse to that specific area of the body that needs to be examined. Due to the RF pulse, protons in that area absorb the energy needed to make them spin in a different direction. This is meant by the resonance of MRI. The RF pulse makes the protons spin at the larmour frequency, in a specific direction. This frequency is found based on the particular tissue being imaged and the strength of the main magnetic field. MRI uses three electromagnetic fields: static field which is a very strong static magnetic field which polarizes the hydrogen nuclei; gradient field which is a weaker timevarying field used for spatial encoding; and a weak radio frequency field for manipulation of the hydrogen nuclei to produce measurable signals, which are collected through radio frequency antenna.

\subsection{The Challenges}

The brain is the anterior most part of the central nervous system. Brain tumour is an intracranial solid neoplasm. Tumours are created by an abnormal and uncontrolled cell division in the brain. It was used axial view of the brain image (2D) from MRI scan because MRI scan is less harmful than CT brain scan. A patient is subjected to different diagnostic methods to determine the cause of the symptoms mentioned by him. Techniques like performing a biopsy, performing imaging, like taking a MRI or CT scan of the brain will be done. In biopsy, pathologists take a specimen of the brain tissue under consideration for checking the presence of tumour. A pathologist looks at the tissue cells under a microscope to check for presence of abnormality. Though biopsy will show the presence of tumour and its pathology, when doctors go for surgery, they must know the tumour extent and the exact location of tumour in the brain, which can be found by taking MRI scan of the patient as MRI doesn't involve the use of harmful radiations when compared to CT scan. Traditional method in hospitals is to segment the medical image under consideration, manually and this depends on how well the physician can perceive the image under consideration to get the required region extracted out, which is made difficult because of minute variations and resemblance between the original and affected biological part in the image. The shortage of radiologists and the large volume of MRI to be analyzed make these readings labor intensive and also cost expensive. [1]

\subsection{Clustering Method}

This is an iterative technique that is used to portion an Image into clusters. In the clustering method number of clusters $\mathrm{K}$ is an input parameter. The main challenge is also the same, because quality of the segmented output depends on the K [12]. Procedure for the clustering is.

1. Pick K cluster centres, either randomly or based on Some heuristic 
2. Assign each pixel in the image to the cluster that minimizes the distance between the pixel and the Cluster centre.

3. Re-compute the cluster centres by averaging all of the pixels in the cluster.

4. Repeat steps 2 and 3 until convergence is attained (E.g. no pixels change clusters).

Clusters can be selected manual y, randomly, or based on Some conditons.Distance between the pixel and cluster centre is calculated by the squared or absolute difference between $n$ a pixel and a cluster centre.

\section{IMAGE SEGMENTATION BY CLUSTERING METHOD}

Clustering can be considered the most important unsupervised learning problem; so, as every other problem of this kind, it deals with finding a structure in a collection of unlabeled data. A definition of clustering could be "the process of organizing objects into groups whose members are similar in some way". A cluster is therefore a collection of objects which are "similar" between them and are "dissimilar" to the objects belonging to other clusters. [2] [3]. [4]. [5].

\subsection{Fuzzy C-Means Clustering}

Fuzzy c-means (FCM) is a method of clustering which allows one piece of data to belong to two or more clusters. [9]. that is it allows the pixels belong to multiple classes with varying degrees of membership. It is based on minimization of the following objective function. The FCM algorithm is one of the most widely used fuzzy clustering algorithms. [6].This technique was originally introduced by Professor Jim Bezdek in 1981. The FCM algorithm attempts to partition a finite collection of elements $X=\left\{X_{1}, x_{2}, \ldots, X_{n}\right\}$ into a collection of c fuzzy clusters with respect to some given criterion. Given a finite set of data, the algorithm returns a list of c cluster centres $\mathrm{V}$, such that

$\mathrm{V}=\mathrm{v}_{\mathrm{i}}, \mathrm{i}=1,2 \ldots \mathrm{c}$

And a partition matrix $U$ such that

$\mathrm{U}=\mathrm{u}_{\mathrm{ij}}, \mathrm{i}=1 \ldots \mathrm{C}, \mathrm{j}=1 \ldots \mathrm{N}$

Where ${ }^{\mathrm{ijj}}$ is a numerical value in $[0,1]$ that tells the degree to which the element $\mathbf{x}_{\mathbf{j}}$ belongs to the i-th cluster.

The following is a linguistic description of the FCM algorithm, which is implemented Fuzzy Logic. The functions that implement this algorithm can be found in the Clustering $\mathrm{m}$ file.

Step 1: Select the number of clusters c $\left(2 \leq_{\mathrm{c}} \leq_{\mathrm{n}}\right)$, exponential weight ${ }^{\mu}\left(1<{ }^{\mu}<\infty\right)$, initial partition matrix $\mathrm{U}^{0}$, and the termination criterion ${ }^{\epsilon}$. Also, set the iteration index 1 to 0 .

Step 2: Calculate the fuzzy cluster centres $\left\{\mathrm{v}^{1} \mid \mathrm{i}=1,2 \ldots \mathrm{c}\right\}$ by using $\mathrm{U}^{\mathrm{l}}$.
Step 3: Calculate the new partition matrix $\mathrm{U}^{\mathrm{l}+1}$ by using $\left\{\mathrm{V}_{i}^{1}\right.$ $\mid \mathrm{i}=1,2, \mathrm{c}\}$.

Step 4: Calculate the new partition matrix ${ }^{\Delta}=\left\|^{\mathrm{U}^{1+1}}-\mathrm{U}^{1}\right\|$ $=\left.\max _{\mathrm{ij}}\right|^{\mathrm{u}_{\mathrm{ij}}{ }^{\mathrm{l}+\mathrm{l}}-\mathrm{u}_{\mathrm{ij}}^{\mathrm{l}} \mid \mathrm{If}}{ }^{\Delta}>^{\epsilon}$, then set $\mathrm{l}=1+1$ and go to step 2. If I $^{\Delta} \epsilon_{\text {, then stop. }}$

We will demonstrate here how to set up and use the clustering functions.[10]

\subsection{K-Means Clustering}

$\mathrm{K}$-means is one of the simplest unsupervised learning algorithms that solve the well known clustering problem. The procedure follows a simple and easy way to classify a given data set through a certain number of clusters fixed a priori. [11]This algorithm aims at minimizing an objective function, in this case a squared error function. [7].

The algorithm is composed of the following steps:

i. Place $\mathrm{K}$ points into the space represented by the objects that are being clustered. These points represent initial group centroid.

ii. Assign each object to the group that has the closest centroid.

iii. When all objects have been assigned, recalculate the positions of the K centroid. Repeat Steps 2 and 3 until the centroid no longer move. This produces a separation of the objects into groups from which the metric to be minimized can be calculated. [8]

\section{PROPOSED ALGORITHM}

The Modified K-mean algorithm is a popular clustering algorithm and has its application in data mining, image segmentation, bioinformatics and many other fields. This algorithm works well with small datasets. In this paper we proposed an algorithm that works well with large datasets. Modified k-mean algorithm avoids getting into locally optimal solution in some degree, and reduces the noise and more the accuracy of cluster.

The algorithm has the following steps.

1. Read the image in to the MATLAB environment using the imread function.

2. Convert the image to $L * a * b$ colour space using the make form and applying form.

3. Calculate the mean in every step.

4. Classifying the colours using $\mathrm{k}$ means clustering label

5. Every pixel in the image using the results from $k$ mean.

6. Create that segmented image by colour using cluster.

\section{EXPERIMENTAL RESULTS} ANALYSIS AND DISCUSSION

The proposed algorithms have been implemented using MATLAB. The performance of various image segmentation approaches are analyzed and discussed. The measurement of image segmentation is difficult to measure. There is no common algorithm for the image segmentation. The statistical measurements could be used to measure the quality of the 
image segmentation. Structural similarity index measures (SSIM), structural content (SC), Mean Squared Error (MSE), and Peak Signal to Noise Ratio (PSNR) are used to evaluate the performance. The detailed description with formulae of SSIM, SC, MSE, PSNR parameters are explained in detail as follows.

\subsection{Structural Similarity Index Measures}

The structural similarity index measures the similarity of the images based on three elements like luminance, contrast, and structure. Images $\mathrm{x}$ and $\mathrm{y}$ the structural similarity index (SSIM) is computed by the formula.[13].

$$
\text { S.S.I.M }(X, Y)=\frac{(2 \mu X \mu Y+C 1)(2 \sigma X Y+C 2)}{(2 \mu X 2+\mu Y 2+C 1)(\sigma 2 X+\sigma 2 Y+C 2)}
$$

Structural similarity index measures have lower computational complexity and superior Performance in image quality assessment task. In structural similarity index measures the information of an image is defined as these attributes that represents the structures of the object in the visual scene, apart from mean intensity and contrasts.SSIM provides surprisgily good image quality prediction performance for a wide variety of image distortions. The maximum structural similarity index measures value is 1 is achieved if And only if $\mathrm{x}$ and $\mathrm{y}$ are in identical. [14].

\subsection{Structural Content}

The value of sc (jaskirat et al.) to a great extent influences the quality of segmented image. Sc measures is given by

$$
\mathrm{SC}=\sum_{i=1}^{m} \sum_{j=1}^{n} \operatorname{in}(i, j) 2 / \sum_{i=1}^{m} \sum_{j=1}^{n} \operatorname{seg}(i, j) 2
$$

Where in $(i, j) 2$ is a the input image and sag $(i, j) 2$ is the target segmented image and $\mathrm{m}$ and $\mathrm{n}$ are matrix row and columns respectively.

A smaller value of structural content (SC) means that the image is of better quality.

\subsection{Means Squared Error}

Mean Square mistake can be approximated in one of many ways to quantify the difference between values implied by an approximate and the true quality being certificated. MSE is a risk function analogous to the expected value of squared error. The MSE is the second moment of error and thus include both the variance of the approximate and its bias. For an $\mathrm{m}^{* \mathrm{n}}$ image the MSE can be calculated as.

$$
\mathrm{MSE}=\frac{1}{m n} \sum_{i=0}^{m-1} \sum_{j=0}^{n-1}[I(i . j)-J(i, j)] 2
$$

Where $I(i, j)$ is input image and $K(i, j)$ is output image. The value of MSE should always be less than PSNR. Lower the value of MSE of an image means less error and high quality of the image. PSNR and MSE are inversely proportional to each other.

\subsection{Peak Signal to Noise Ratio}

The Peak Signal to Noise Ratio (PSNR) is the proportion between maximum attaible powers and corrupting noise that influence likeness of image. PSNR is usually précised as decibel scale. The PSNR is usually used as measure of quality rebuilding of image. The signal in this case is original data and the noise is the error imported. High value of PSNR signifies the big Quality of image.

It is explain via the Mean Square Error (MSE) and analogous deformity metric, the Peak Signal to Noise Ratio. Here Max is maximum pixel value of image when pixel is represented by using 8 bits per sample. This is 255 bar colour image with three RGB value per pixel.

$$
\begin{gathered}
\text { PSNR }=10 \cdot \log 10\left(\frac{M A X^{2} I}{M S E}\right) \\
=20 \cdot \log 10\left(\frac{M A X I}{\sqrt{M S E}}\right) \\
=20 \cdot \log 10(M A X I)-10 \log 10(M S E)
\end{gathered}
$$

Higher the PSNR values, better the quality of image. If PSNR value is above 30 , that means the output has hundred per cent image clarity. The unit of PSNR is $\mathrm{dB}$ (decibel). It takes from 0 to infinity. The PSNR section calculates the peak signal-tonoise proportion in decibels, between two Images. This percentage is often used as a feature measurement between the original and a compact image. The higher the PSNR, the better the quality of the compact or constructed image.

The input and output images are shows here, input images shows in the figure (a) and output images shows Figure (b) Figure (c) and Figure (d) depicts the segmentation of MRI images using $\mathrm{K}$ means clustering and fuzzy $\mathrm{c}$ means clustering respectively. Through the visual identification, it can observe that the edges of segmented MRI images are well preserved in the modified $\mathrm{k}$ means clustering techniques.

Figure 1. (a) Original image (b) C-Means Images (c) KMeans Images (d) Modified K- Means Images or Proposed Method.

(a)
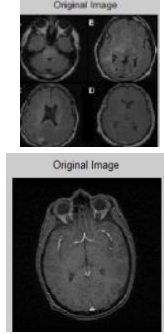

(b)

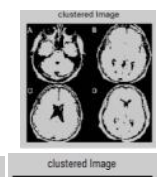

(c)

(d)

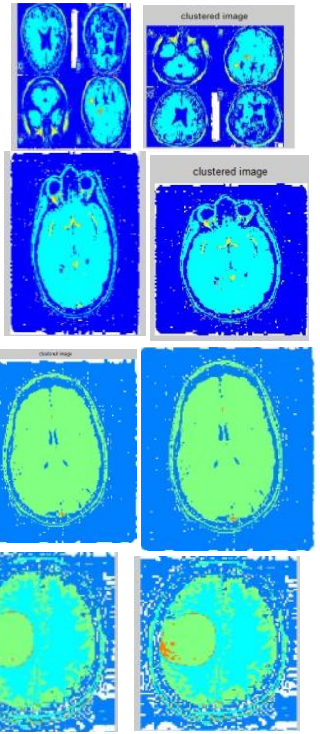

Figure1. Image Segmentation Using Clustering 
Table 1. Performance Evaluation

\begin{tabular}{|c|c|c|c|c|c|}
\hline $\begin{array}{c}\text { IMAG } \\
\text { ES }\end{array}$ & $\begin{array}{c}\text { MET } \\
\text { HOD } \\
\text { S }\end{array}$ & SC & SSIM & MSE & PSNR \\
\hline \multirow[t]{3}{*}{ Image 1} & FCM & $\begin{array}{l}6.350 \\
1\end{array}$ & 0.3877 & 8.4964 & 8.8385 \\
\hline & KCM & $\begin{array}{l}4.746 \\
6\end{array}$ & 0.3377 & 6.6878 & 9.8715 \\
\hline & $\begin{array}{l}\text { MKC } \\
\text { M }\end{array}$ & $\begin{array}{l}1.227 \\
9\end{array}$ & 0.6128 & 549.53 & 20.730 \\
\hline \multirow[t]{3}{*}{ Image 2} & FCM & $\begin{array}{l}6.264 \\
8\end{array}$ & 0.4182 & 5.9053 & 10.418 \\
\hline & $\mathrm{KCM}$ & $\begin{array}{l}5.863 \\
7\end{array}$ & 0.3077 & 6.4734 & 10.019 \\
\hline & $\begin{array}{l}\text { MKC } \\
\text { M }\end{array}$ & $\begin{array}{l}1.229 \\
1\end{array}$ & 0.5229 & 496.29 & 21.173 \\
\hline \multirow[t]{3}{*}{ Image 3} & FCM & $\begin{array}{l}8.787 \\
9\end{array}$ & 0.4500 & 6.9635 & 9.7025 \\
\hline & $\mathrm{KCM}$ & $\begin{array}{l}13.45 \\
3\end{array}$ & 0.2066 & 1.4650 & 6.4723 \\
\hline & $\begin{array}{l}\text { MKC } \\
\text { M }\end{array}$ & $\begin{array}{l}1.239 \\
6\end{array}$ & 0.5277 & $\begin{array}{l}365.05 \\
0\end{array}$ & $\begin{array}{l}22.507 \\
3\end{array}$ \\
\hline \multirow[t]{3}{*}{ Image 4} & FCM & $\begin{array}{l}1.958 \\
7\end{array}$ & 0.5294 & 3.3034 & 12.941 \\
\hline & $\mathrm{KCM}$ & $\begin{array}{l}2.821 \\
3\end{array}$ & 0.2296 & 1.3006 & 6.9893 \\
\hline & $\begin{array}{l}\text { MKC } \\
M\end{array}$ & $\begin{array}{l}1.035 \\
4\end{array}$ & 0.5297 & $\begin{array}{l}590.48 \\
0\end{array}$ & $\begin{array}{l}20.418 \\
7\end{array}$ \\
\hline
\end{tabular}

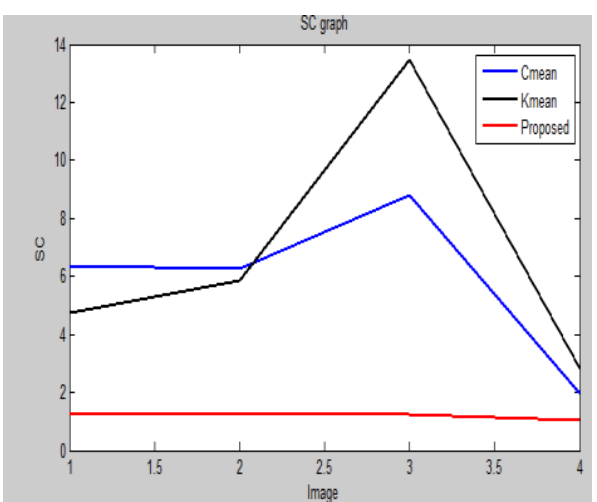

(a)

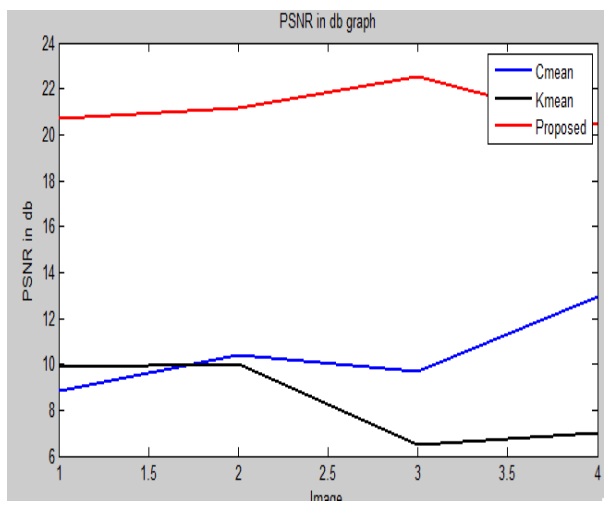

(b)

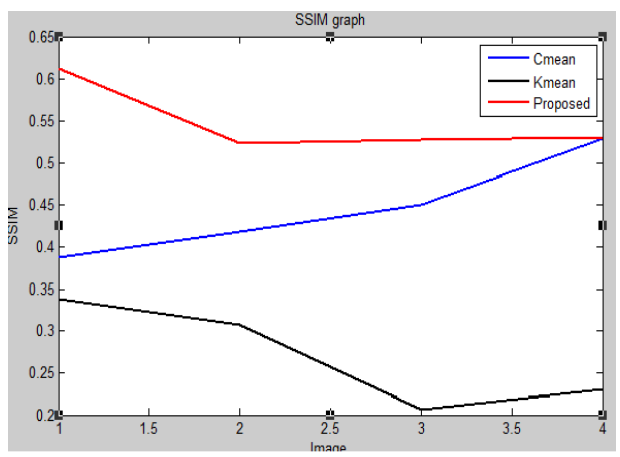

(c)

Figure.3 Graph Analysis (a) SC (b) PSNR (c) SSIM

\section{CONCLUSION}

The unsupervised method i.e. cluster based algorithms were proposed for image segmentation. The clustering techniques such as $\mathrm{k}$ means, fuzzy c mean, were tested in different images. The performance of proposed algorithms is measured using segmentation parameters SC, SSIM, MSE, and PSNR. The computational results showed that the $\mathrm{K}$ means image segmentation has less accuracy but it provide poor result. The $\mathrm{k}$ means algorithm takes minimum numbers of iterations compare to $\mathrm{C}$ means. The $\mathrm{KCM}$ consume more time and provide good result where as the FCM algorithm consume less time compare to $\mathrm{k}$ means and provide good result. Therefore form the computational results conclude that the proposed algorithms the modified $\mathrm{k}$ means algorithm performed better than others in terms of performance accuracy and better convergence rate.

\section{REFERENCES}

[1] M.Masroor Ahmed, Dzulkifli Bin Mohamad, "Segmentation of Brain MR Images for Tumor and combining by $\mathrm{k}$ means clustering International Journal of Image Processing, vol. 2 , no. 1, pp 27-34,2008

[2] U.M. Fayyad, G. Piatetsky-Shapiro, P. Smyth, R. Uthurusamy, Advances in Knowledge Discovery and Data Mining,AAAI/MIT Press (1996)

[3] M.N. Murty, A.K. Jain, P.J. Flynn, and Data clustering: a review, ACM Computes. Survey. 31(3) (1999) 264-323

[4] A.K. Jain, R.C. Dubes, Algorithms for Clustering Data,Prentice Hall, Englewood Cliffs, NJ(1988)

[5] R.T. Ng, J. Han, Efficient and effective clustering methods for spatial data mining, in: Proceedings of the Twentieth International Conference on Very Large Databases, Santiago, Chile(1994) 144-155620

[6] Xiuyun Li, Jie Yang, Qing Wang, Jinjin Fan, Pang Liu,2010 Research and Application of Improved Kmeans Algorithm Based on Fuzzy Feature Selection.

[7] Shehroz S. Khan, Amir Ahmad,Cluster center initialization algorithm for K-means clustering, Pattern Recognition Letters 25 (2004) 1293-1302

[8] Bradley, P.S., Fayyad, U.M., Refining initial points for K Means clustering. In: Sharlik, J. (Ed.), Proc. 15th Internat. Conf. on Machine Learning (ICML98). Morgan Kaufmann, San Francisco, CA,(1998) 91-99 621

[9] K.S.Ravichandran and B. Acanthi," Color Skin Segmentation Using K-Means" Cluster International Journal of Computational and Applied Mathematics 
ISSN 1819-4966 Volume 4 Number 2 (2009), pp. 153157

[10] Jude hemanth.D, D.Selvathi and J.Anitha,"Effective Fuzzy Clustering Algorithm for Abnormal MR Brain Image Segmentation", International/Advance Computing Conference (IACC 2009), IEEE, 2009.

[11] Sorin Istrail, "An Overview of Clustering Methods", With Applications to Bioinformatics.

[12] Dzung L. Pham, ChenyangXu, and Jer y L. Prince Current Methods In Medical Image Segmentation
Department of Electrical and Computer Engine ring, The Johns Hopkins University, An u. Rev. Biomed. Eng. 20 0. $02: 315 \tilde{n} 37$

[13] Zhou Wang, Alan Conrad Bovik., et.al. Image Quality Assessment: From Error Visibility to Structural Similarityll IEEE Transactions on Image processing, vol. 13, no.4, April 2004.

[14] Ahmet M. Eskicioglu and Paul S. Fisher Image Quality Measures and their performancell IEEE Transactions on Communications, Vol.43, No.12, December 1995. 